\title{
Serum periostin levels in asthma patients in relation to omalizumab therapy and presence of chronic rhinosinusitis with nasal polyps
}

\author{
Jakub Novosad¹, Irena Krčmová1, Vladimír Bartoš², Marcela Drahošová1, Petr Vaník³, Olga Růžičková-Kirchnerová4, \\ Milan Teřl ${ }^{4}$, Jan Krejsek ${ }^{1}$ \\ ${ }^{1}$ Institute of Clinical Immunology and Allergy, University Hospital in Hradec Králové, Faculty of Medicine, Charles University in Prague, \\ Czech Republic \\ ${ }^{2}$ Department of Pulmonary Medicine, University Hospital in Hradec Králové, Faculty of Medicine, Charles University in Prague, \\ Czech Republic \\ 3Department of Respiratory Diseases, Hospital in České Budějovice, Faculty of Medicine in Pilsen, Charles University in Prague, \\ Czech Republic \\ ${ }^{4}$ Department of Pulmonary Medicine, University Hospital in Pilsen, Faculty of Medicine, Charles University in Prague, Czech Republic \\ Adv Dermatol Allergol 2020; XXXVII (2): 240-249 \\ DOI: https://doi.org/10.5114/ada.2020.94842
}

\begin{abstract}
Introduction: The serum periostin level is a promising biomarker of type 2- high inflammation pattern of bronchial asthma. It has been proven that serum periostin levels decrease in response to systemic and inhaled corticosteroid (ICS) therapy. However, we have only limited knowledge about changes in serum periostin levels reflecting omalizumab (OMA) treatment and other variables, such as chronic rhinosinusitis with nasal polyps (CRSwNP).

Aim: To critically appraise clinically relevant parameters influencing periostin levels in asthma patients.

Material and methods: A pilot, cross-sectional, observational study to assess serum periostin levels of 48 asthma patients ( 38 treated by conventional therapy comprising ICS and 10 treated by ICS and OMA as an add-on therapy) with respect to asthma clinical traits, comorbidities and to other biomarkers of type 2-high asthma phenotype (total IgE, absolute and relative eosinophil count, eosinophilic cationic protein (ECP) and a fraction of exhaled NO (FeNO)). Results: Serum periostin correlates with total IgE levels (Spearman rho $=0.364, p=0.025$ ) in a subgroup of conventionally treated patients, and with eosinophil count (Spearman rho $=0.401, p=0.021$ ) in a subgroup of patients with concurrent CRSwNP. Serum periostin levels were decreased in omalizumab-treated patients in comparison to conventionally treated patients $(p=0.025)$. This effect was remarkably apparent only if CRSwNP was not present $(p=0.005)$. Conversely, we measured elevated periostin levels in OMA-treated patients with concurrent CRSwNP $(p=0.017)$.

Conclusions: Serum periostin production is significantly associated with treatment modality (omalizumab vs. conventional) and presence of CRSwNP. These variables need to be taken into account to interpret periostin levels accurately.
\end{abstract}

Key words: periostin, asthma phenotype, omalizumab, chronic rhinosinusitis with nasal polyps.

\section{Introduction}

Periostin is a 90kDa matricellular protein, discovered in 1993 [1] and fully characterized in 1999 [2]. It has been demonstrated that periostin plays an important role in the pathogenesis of allergic inflammation including asthma and both tissue remodelling [3] and mucus production, with special emphasis on pulmonary pathology [4].
In 2009 Woodruff et al. showed that periostin expression is associated with type 2-high inflammation pattern in asthma [5]. Periostin has been observed in the thickened basement membrane [6] as well as in serum of asthmatic patients with eosinophilic airway inflammation, regardless of atopy [7].

Moreover, the serum periostin level has demonstrated its usefulness to identify the most eligible patients

Address for correspondence: Irena Krčmová MD, PhD, Institute of Clinical Immunology and Allergy, University Hospital, 581 Sokolská St, 50005 Hradec Králové, Czech Republic, phone: +42 495833771, e-mail: irena.krcmova@fnhk.cz

Received: 14.09.2018, accepted: 19.10.2018. 
for treatment by inhaled corticosteroids (ICS) [8], by lebrikizumab [9] and at least in part by omalizumab (OMA) $[10,11]$. Encouraging results instigated more thorough investigation in this field extending measurement of periostin serum levels in clinical practice to improve not only the asthma diagnostics and classification but also the monitoring of treatment effects $[12,13]$ in a disease phenotype-specific approach. Currently, we have evidence that serum periostin levels in asthma patients decrease in response to the therapy by systemic or inhaled corticosteroids [14] as well as to OMA [15]. Its production is influenced by some comorbidities, such as allergic rhinitis, chronic rhinosinusitis [16, 17], atopic dermatitis [18] or aspirin intolerance. However, the clinical relevance of the significance of mutual interactions of factors mentioned above on periostin production still remains to be clarified.

The Czech societies for allergy and clinical immunology and for respiratory medicine have implemented this pheno-/endotypic approach to management of bronchial asthma (eosinophilic allergic asthma/eosinophilic non-allergic asthma/non-eosinophilic asthma) into the routine [19]. It is broadly accepted that eosinophilic inflammation is being recognized as a treatable trait of asthma and therefore its assessment is of big interest. Moreover, today we have constantly broadened the range of phenotype-specific biological treatments targeted mainly at eosinophilic inflammation (monoclonal antibodies antiIgE, anti-interleukin-5 (IL-5/IL-5R), anti-IL-4R (IL-4 receptor), anti-thymic stromal lymphopoietin - TSLP) hopefully in the near future [20].

Nonetheless, current research revealed that there are substantial differences between at least two different representatives of type 2 - high, eosinophilic phenotypes. Schematically, either eosinophilic allergic (Th2-high, orchestrated mainly by Th2 lymphocytes with leading signalization by IL-4 and IL-13) or eosinophilic non-allergic (ILC-high, driven mainly by innate lymphoid cells 2 with dominating IL-5 and IL-13 signalization) [21]. It seems to be reasonable and meaningful to discern between these two distinct entities in clinical practice because of their distinct molecular pathogenesis, different clinical course, prognosis and treatment responsiveness [22]. It is very likely that to tackle these diagnostic obstacles, we will need not a single biomarker, but rather a panel of biomarkers to define particular biological processes or inflammation patterns, in particular for asthma patients. Serum periostin could be one of them.

Nowadays, we measure the eosinophil count in peripheral blood or sputum, total IgE or eosinophilic cationic protein (ECP) in serum or the fraction of nitric oxide in exhaled breath (FeNO) in routine practice. Despite a seemingly sufficient range of available biomarkers including periostin, all of them display only a weak mutual crosscorrelation and are not easily interchangeable [23] since they reflect slightly different immunological processes.
Unfortunately, in contrast to other type 2-high inflammation associated biomarkers, the periostin laboratory samples processing and assay workflow lack standardization and do not meet the required standards for diagnostic accuracy yet [24]. Thus, interpretation or direct comparison of serum periostin levels coming from studies using different assays (either electrochemiluminescence or enzyme-linked immunosorbent (ELISA) assays) is difficult [25]. That is probably one of the principal reasons why periostin is still not widely used in routine, despite its low cost and readily measurement.

\section{Aim}

The aim of our study was to introduce the serum periostin measurement into broader clinical practice and to investigate potential pitfalls of results interpretation with respect to treatment and comorbidities of bronchial asthma, as well as to critically appraise its clinical usefulness.

\section{Material and methods}

\section{Study objectives and design}

The aim of our pilot, cross-sectional, observational study was a critical appraisal of an effect of treatment modality (conventional therapy vs. OMA therapy) and comorbidities on serum periostin levels, with respect to anthropometry, pulmonary functions and other clinical asthma traits in asthma patients. The study had a simple independent design with a single visit measurement of routinely assessed blood biomarkers (including periostin, eosinophil count, total IgE and eosinophilic-cationic protein (ECP)), pulmonary function tests, FeNO, clinical examination and verification of patient's personal history. Data were collected and analysed throughout 2015. The study was approved by the local ethics committee of the University Hospital in Hradec Králové (reference number: 201211 S19P).

\section{Study sample characteristics and treatment}

The study group consisted of 48 asthma patients without any concomitant pulmonary pathology (19 males/29 females, mean age \pm SD: $47.7 \pm 12.5)$, mean body-mass index (BMI) $27.3 \pm 5.62 \mathrm{~kg} / \mathrm{m}^{2}$ ), diagnosed, characterized and treated according to the national standards [19] with observance of international guidelines [26] at the Institute of Clinical Immunology and Allergy, University Hospital, Hradec Králové, Czech Republic. All patients were treated with conventional asthma therapy (encompassing inhaled corticosteroids (ICS) and longacting $\beta$-agonists (LABA)), 10 of them received OMA as an add-on therapy. All OMA-treated patients were good responders to therapy [27]. Nine patients used oral corticosteroids (7 in conventional and 2 in the OMA arm). For more detailed study sample characteristics see Table 1. 


\section{Laboratory procedures}

Serum periostin levels were measured using ELISA (BioVendor, Czech Republic) by ELISA reader Dynatech MRX (Microplate Reader). All other biomarkers of type 2-high asthma endotype (total IgE, absolute eosinophil count (AEC), relative eosinophil count (eos\%), eosinophilic cationic protein (ECP)) were measured according to international standards in a certified laboratory of the Institute of Clinical Immunology and Allergy, University Hospital, Hradec Králové, Czech Republic. Since OMA creates small, biologically inactive complexes with IgE molecules, the trough serum levels of total IgE during OMA treatment are biased [28]. Therefore, in the omalizumab- treated patients' group, we analysed the baseline total IgE levels (data collected retrospectively from measurements before OMA treatment initiation), not the trough IgE levels (in contrast to measurements of all other biomarkers).

\section{Clinical procedures}

All patients underwent a routine clinical examination including personal medical history, physical examination including weight, height, BMI calculation, pulmonary function tests (spirometry with maximal vital capacity VCmax, $\mathrm{FEV}_{1}$ in $\mathrm{ml}$ and $\%$ of predicted and $\mathrm{FEV}_{1} / \mathrm{VCmax}$ ratio calculation, airway obstruction defined as $\mathrm{FEV}_{1} /$

Table 1. Study sample characteristics. Basic demography and anthropometry of the study population and subpopulations according to the treatment modality and presence of CRSwNP (Pearson $\chi^{2}$ and Mann-Whitney $U$ test)

\begin{tabular}{|c|c|c|c|c|c|c|c|c|}
\hline \multirow{2}{*}{\multicolumn{2}{|c|}{ Parameter }} & \multirow[t]{2}{*}{ Total } & \multicolumn{3}{|c|}{ Therapy } & \multicolumn{3}{|c|}{ CRSwNP } \\
\hline & & & $\begin{array}{l}\text { Conv. } \\
\text { therapy }\end{array}$ & OMA & $P$-value & No & Yes & $P$-value \\
\hline \multicolumn{2}{|c|}{ Sample size, $n$} & 48 & 38 & 10 & - & 33 & 15 & - \\
\hline \multicolumn{2}{|c|}{ Sex (male/female) } & $19 / 29$ & $14 / 24$ & $5 / 5$ & $0.449(\mathrm{a})$ & $11 / 22$ & $8 / 7$ & $0.189(\mathrm{a})$ \\
\hline \multicolumn{2}{|c|}{ Age (mean/SD) [years] } & $47.7 / 12.5$ & $49 / 13$ & $44 / 10$ & 0.309 (b) & $47 / 13$ & $50 / 12$ & $0.338(b)$ \\
\hline \multicolumn{2}{|c|}{ Height (mean/SD) [cm] } & $170 / 10$ & $170 / 10$ & $170 / 10$ & 0.851 (b) & $169 / 9$ & $170 / 12$ & $0.577(b)$ \\
\hline \multicolumn{2}{|c|}{ Weight (mean/SD) [kg] } & $79 / 20$ & $78 / 21$ & $83 / 17$ & $0.445(b)$ & $78 / 22$ & $82 / 13$ & $0.225(b)$ \\
\hline \multicolumn{2}{|c|}{$\mathrm{BMI}(\mathrm{mean} / \mathrm{SD})\left[\mathrm{kg} / \mathrm{m}^{2}\right]$} & $27.3 / 5.62$ & $26.9 / 5.57$ & $28.7 / 5.9$ & $0.430(b)$ & $26.8 / 6.13$ & $28.3 / 4.29$ & $0.161(b)$ \\
\hline \multicolumn{2}{|c|}{ Obesity (yes/no) } & $10 / 38$ & $6 / 32$ & $4 / 6$ & $0.093(\mathrm{a})$ & $7 / 26$ & $3 / 12$ & $0.924(\mathrm{a})$ \\
\hline \multicolumn{2}{|c|}{ Exacerbation rate (mean/min./max.) } & $1.91 / 0 / 12$ & $1.91 / 0 / 12$ & $1.90 / 0 / 10$ & $0.742(b)$ & $1.41 / 0 / 10$ & $2.93 / 0 / 12$ & $0.093(b)$ \\
\hline \multicolumn{2}{|c|}{$\begin{array}{l}\text { OMA therapy duration (mean/min./ } \\
\text { max.) [months] }\end{array}$} & $26 / 3 / 60$ & 0 & $26 / 3 / 60$ & - & $5.15 / 0 / 48$ & $5.67 / 0 / 60$ & $0.938(b)$ \\
\hline \multicolumn{2}{|c|}{ Comorbidities in general (yes/no) } & $46 / 2$ & $37 / 1$ & $9 / 1$ & $0.299(\mathrm{a})$ & $32 / 1$ & $14 / 1$ & $0.559(a)$ \\
\hline \multicolumn{2}{|c|}{ Smoking status (yes/no) } & $2 / 46$ & $2 / 36$ & $0 / 10$ & $0.459(\mathrm{a})$ & $1 / 32$ & $1 / 14$ & $0.559(a)$ \\
\hline \multicolumn{2}{|c|}{ Airway obstruction (yes/no) } & $30 / 18$ & $25 / 13$ & $5 / 5$ & $0.359(a)$ & $20 / 13$ & $10 / 5$ & $0.688(a)$ \\
\hline \multicolumn{2}{|c|}{ AERD (yes/no) } & $9 / 39$ & $6 / 32$ & $3 / 7$ & $0.306(a)$ & $3 / 30$ & $6 / 9$ & $0.011(a)$ \\
\hline \multicolumn{2}{|c|}{ Atopy status (yes/no) } & $39 / 9$ & $29 / 9$ & $10 / 0$ & $0.088(\mathrm{a})$ & $27 / 6$ & $12 / 3$ & $0.881(\mathrm{a})$ \\
\hline \multicolumn{2}{|c|}{ Atopic eczema (yes/no) } & $13 / 35$ & $9 / 29$ & $4 / 6$ & $0.302(\mathrm{a})$ & $12 / 21$ & $1 / 14$ & $0.032(a)$ \\
\hline \multicolumn{2}{|c|}{ Allergen exposure (yes/no) } & $15 / 33$ & $14 / 24$ & $1 / 9$ & $0.103(a)$ & $11 / 22$ & $4 / 11$ & $0.644(a)$ \\
\hline \multirow{4}{*}{$\begin{array}{l}\text { Asthma } \\
\text { severity }(n)\end{array}$} & Mild & 4 & 4 & 0 & $0.041(\mathrm{a})$ & 4 & 0 & $0.428(a)$ \\
\hline & Moderate & 9 & 9 & 0 & & 7 & 2 & \\
\hline & Severe & 6 & 6 & 0 & & 4 & 2 & \\
\hline & Severe Refractory & 29 & 19 & 10 & & 18 & 11 & \\
\hline \multirow{4}{*}{$\begin{array}{l}\text { Asthma } \\
\text { control }(n)\end{array}$} & Controlled & 7 & 6 & 1 & $0.967(a)$ & 7 & 0 & $0.068(a)$ \\
\hline & Partly controlled & 27 & 21 & 6 & & 19 & 8 & \\
\hline & Uncontrolled & 10 & 8 & 2 & & 6 & 4 & \\
\hline & Exacerbation & 4 & 3 & 1 & & 1 & 3 & \\
\hline \multirow{3}{*}{$\begin{array}{l}\text { Asthma } \\
\text { phenotype } \\
(n)\end{array}$} & Eosinophilic allergic & 37 & 27 & 10 & $0.153(a)$ & 25 & 12 & $0.097(a)$ \\
\hline & Eosinophilic non-allergic & 5 & 5 & 0 & & 2 & 3 & \\
\hline & Neutrophilic & 6 & 6 & 0 & & 6 & 0 & \\
\hline
\end{tabular}

(a) Pearson $\chi^{2}$, (b) Mann-Whitney $U$ test. 
VCmax $<5^{\text {th }}$ percentile of predicted [29]) and FeNO measurement. Pulmonary function tests were performed using Jaeger MasterScreen (CareFusion GmbH, Hoechberg, Germany) spirometer and FeNO was assessed using HypAir FeNO (Medisoft, Sorinnes, Belgium) electrochemical analyzer. Spirometry and FeNO measurement fulfilled all standard requirements [30, 31]. Other specialized examinations (including examination by a dermatologist and an ear-nose-throat specialist) were performed by appropriate specialists.

\section{Definition of asthma phenotypes and comorbidities}

Asthma diagnosis and control was established according to the international guidelines [26]. Additionally, the asthma phenotype (eosinophilic-allergic, eosinophilic-non-allergic, non-eosinophilic) was defined in line with the national recommendations [19], with respect to the patient's eosinophil count in peripheral blood, serum ECP and FeNO level and allergy/atopy status. Atopy was defined as a positive skin prick test and/or positivity of serum specific lgE to a clinically relevant allergen. Allergen exposure during the study visit was taken into account. The number of severe exacerbations [32] during the previous year was calculated.

Extrapulmonary comorbidities of asthma were assessed - atopic eczema [33], chronic rhinosinusitis with or without nasal polyps - CRSwNP/CRSsNP [34], smoking status (two categories: current smokers / ex-smokers or never smokers) and obesity (defined as BMI > $30 \mathrm{~kg} / \mathrm{m}^{2}$ ). Previously diagnosed aspirin-exacerbated respiratory disease (AERD) by a nasal provocation test with lysineacetylsalicylic acid (L-ASA) [35] (performed at our clinical laboratory of pulmonary and nasal functions, using rhinomanometer Jaeger MasterScreen, CareFusion GmbH, Hoechberg, Germany) was considered. Study sample characteristics are detailed in Table 1.

\section{Study approval}

The ethics committee, University Hospital Hradec Králové, 23 October 2012. Reference number: 201211 S19P.

\section{Statistical analysis}

Descriptive statistics were expressed by the mean and standard deviation or by the median and interquartile range $(I Q R)$ in cases of non-normal data distribution. Cross-correlation was calculated and expressed by the Spearman Rho correlation coefficient; independent groups were compared using the Mann-Whitney $U$ test or Kruskal-Wallis H test, Bonferroni correction was applied where applicable. Frequencies were treated by $\chi^{2}$ test or Fisher's exact test where appropriate, in cases of binary outcome variable, the logistic regression was applied. Factorial ANOVA designs were treated as general linear models, $p<0.05$ was recognized as statistically significant. Data were analysed by IBM SPSS Statistics for Windows, Version 22.0. Armonk, NY: IBM Corp.

\section{Results}

\section{Treatment characteristics}

The majority of patients had been treated for asthma for more than 5 years and suffered from severe $(n=6$, GINA, step 4) or severe refractory asthma ( $n=29$, GINA, step 5) [26]. Type 2-high (eosinophilic inflammation phenotype was confirmed in 42 patients $(n=37$ allergic, $n=5$ non-allergic) [19]. The median month dose of OMA in 10 patients was $450 \mathrm{mg}$ (range: 150-1200 mg), the average treatment duration was 26 months (range: 360 months), and the median cumulative dose was 10350 mg. All OMA-treated patients used maintenance therapy comprising ICS and LABA. Nine patients used oral corticosteroids ( 7 in conventional and 2 in the OMA arm, $p=0.909)$. Treatment characteristics are detailed in Table 1.

\section{Study sample subgroups' characteristics according} to treatment

There were no significant differences between conventionally or OMA-treated patients with respect to frequencies of sex, mean age, height, weight, BMI and presence of obesity. Moreover, both subgroups had a similar exacerbation rate and frequencies of relevant comorbidities in general, smoking status, airway obstruction, AERD, atopy status, atopic eczema and history of exposure to allergens. There were insignificant differences in asthma control and asthma phenotypes. Asthma was in most cases under partial control $(n=27$ in the whole sample, $n=21$ in conventionally treated patients, $n=6$ in OMA-treated patients) and the most frequent clinical phenotype was recognized as eosinophilic allergic $(n=$ 37 in the whole sample, $n=27$ in conventionally treated patients and in all ( $n=10)$ OMA-treated patients). All OMA-treated patients suffered from severe refractory asthma, while the conventionally treated patients fulfilled criteria for mild $(n=4)$, moderate $(n=9)$, severe $(n=6)$ and mostly severe refractory asthma $(n=19)$. Given the above, the frequencies of particular asthma severity grades were the only statistically significant difference between conventionally and OMA-treated patients. Data are reviewed in Table 1.

\section{Study sample subgroup characteristics' according to the presence of CRSwNP}

Chronic rhinosinusitis with nasal polyps (CRSwNP) has been present in 15 patients, while in 33 was missing (patients had either no chronic rhinosinusitis, $n=5$, or suffered from rhinosinusitis without nasal polyps, $n=28$ ). There has been a similar proportion of frequen- 
cies of treatment modality across all patients with respect to the presence of CRSWNP ( $p=0.924)$ (Table 2 A).

Among patients with CRSwNP, 12 had eosinophilic allergic and 3 eosinophilic non-allergic asthma. All patients suffering from non-eosinophilic asthma were without any concomitant CRSwNP. Despite this disproportion, the overall frequencies of particular asthma phenotypes revealed insignificant differences ( $p=0.097$ ). In contrast, to study treatment subgroups, there were insignificant differences even in frequencies of asthma severity and asthma control levels ( $p=0.428 ; p=0.068$ ) (Table 1$)$. However, we have registered a statistically significant difference in frequencies of atopic eczema $(p=0.032)$ and AERD ( $p=0.011)$ with respect to the presence of CRSwNP (Tables 2 B, C). The presence of CRSwNP significantly heightened the odd of AERD on our study sample (OR = 6.67, $p=0.018$; 95\% Cl: 1.38-32.15) (Table 2 C).

We also assessed the asthma severity frequencies in conventionally treated patients with respect to the presence of CRSWNP and found no statistically significant association $(p=0.360)$ (Table $2 \mathrm{D})$.

\section{Periostin levels in study subgroups}

Serum periostin levels were generally decreased in OMA-treated asthma patients in contrast to conventionally treated patients ( $p=0.025)$, regardless of OMA monthly or cumulative dose and treatment duration. Moreover, periostin was the only parameter differing significantly between these two groups in contrast to other biomarkers of type 2-high eosinophilic inflammation pattern (FeNO, relative or absolute eosinophil count, ECP and total IgE levels). Results are summarized in Table 3.
We found an insignificant overall effect of atopic eczema ( $p=0.935), \operatorname{CRSsNP}(p=0.140), \operatorname{CRSwNP}(p=$ $0.087)$, obesity $(p=0.526)$ and $\operatorname{AERD}(p=0.822)$ on periostin levels in the whole study sample. Moreover, there was no significant effect of asthma severity on periostin levels in conventionally treated patient subgroup (Kruskal-Wallis test; $p=0.340$ ), (all patients in the OMA subgroup suffered from severe refractory asthma).

The deeper analysis of the effect of comorbidities on periostin levels unravelled a significant interaction between the therapy modality and CRSWNP. In the subgroup of patients treated by OMA $(n=10)$, we observed a significant elevation of periostin levels in those suffering from CRSwNP $(n=3)$, in comparison to OMA-treated patients without concurrent CRSWNP ( $n=7, p=0.017)$. The overall effect of CRSwNP was negligible in conventionally treated patients $(p=0.530)$ as well as in the whole study group ( $p=0.087$ ) but reached a statistical significance in the subgroup of patients treated by OMA $(p=0.017)$. Conversely, the effect of treatment modality on serum periostin was maxed out in 33 patients without a concurrent CRSwNP $(p=0.005)$ in contrast to those with CRSwNP $(n=15 ; p=0.386)$. We did not observe any other significant interaction between other comorbidities and treatment. Subgroup analysis is presented in Figure 1.

\section{Correlation analysis}

Surprisingly, serum periostin expressed an insignificant cross-correlation with the other type 2-high biomarkers in the whole study sample. However, we observed a weak but statistically significant correlation

Table 2. Contingency tables. A - CRSwNP and treatment modality contingency table Pearson $\chi^{2} ; p=0.924, \mathrm{~B}-\mathrm{CRSwNP}$ and atopic eczema contingency table, Pearson $\chi^{2} ; p=0.032$, likelihood ratio $=5.463, p=0.019, \mathrm{OR}=0.125(p=0.058 ; 95 \% \mathrm{Cl}$ : $0.015-1.072), C-C R S w N P$ and AERD contingency table, Pearson $\chi^{2} ; p=0.011$, likelihood ratio $=6.031 ; p=0.014, \mathrm{OR}=6.67$ $(p=0.018 ; 95 \% \mathrm{Cl}: 1.382-32.154), \mathrm{D}-\mathrm{CRSwNP}$ and asthma severity in conventionally treated patients, Pearson $\chi^{2} ; p=0.360$

A

\begin{tabular}{lcccc}
\hline Parameter & & \multicolumn{2}{c}{ Treatment $(n)$} & Total \\
\cline { 2 - 4 } & & Conventional & Omalizumab & \\
\hline CRSwNP $(n)$ & No & 26 & 7 & 33 \\
\cline { 2 - 5 } & Yes & 12 & 3 & 15 \\
\hline Total & & 38 & 10 & 48 \\
\hline
\end{tabular}

Pearson $\chi^{2}, p=0.924$.

\section{C}

\begin{tabular}{lcccc}
\hline Parameter & & \multicolumn{2}{c}{ AERD $(n)$} & \multirow{2}{*}{ Total } \\
\cline { 2 - 4 } & & No & Yes & \\
\hline CRSWNP $(n)$ & No & 30 & 3 & 33 \\
\cline { 2 - 4 } & Yes & 9 & 6 & 15 \\
\hline Total & & 39 & 9 & 48 \\
\hline
\end{tabular}

Pearson $\chi^{2}, p=0.011$, likelihood ratio $=6.031, p=0.014, O R=6.67(p=0.018$; 95\% Cl: 1.382-32.154).
B

\begin{tabular}{lcccc}
\hline \multirow{2}{*}{ Parameter } & & \multicolumn{2}{c}{ Atopic eczema $(n)$} & \multirow{2}{*}{ Total } \\
\cline { 2 - 4 } & & No & Yes & \\
\hline CRSwNP $(n)$ & No & 21 & 12 & 33 \\
\cline { 2 - 4 } & Yes & 14 & 1 & 15 \\
\hline Total & 35 & 13 & 48 \\
\hline
\end{tabular}

Pearson $\chi^{2}, p=0.032$, likelihood ratio $=5.463, p=0.019, O R=0.125$, ( $p=0.058 ; 95 \%$ Cl: 0.015-1.072).

D

\begin{tabular}{lcccccc}
\hline Parameter & \multicolumn{4}{c}{ Asthma severity $(n)$} & \multirow{2}{*}{ Total } \\
\cline { 2 - 6 } & & Mild & Moderate & Severe & $\begin{array}{c}\text { Severe } \\
\text { refractory }\end{array}$ \\
\hline $\begin{array}{l}\text { CRSwNP } \\
(n)\end{array}$ & No & 4 & 7 & 4 & 11 & 26 \\
\cline { 2 - 6 } & Yes & 0 & 2 & 2 & 8 & 12 \\
\hline Total & 4 & 9 & 6 & 19 & 38 \\
\hline
\end{tabular}

Pearson $\chi^{2}, p=0.360$. 
A
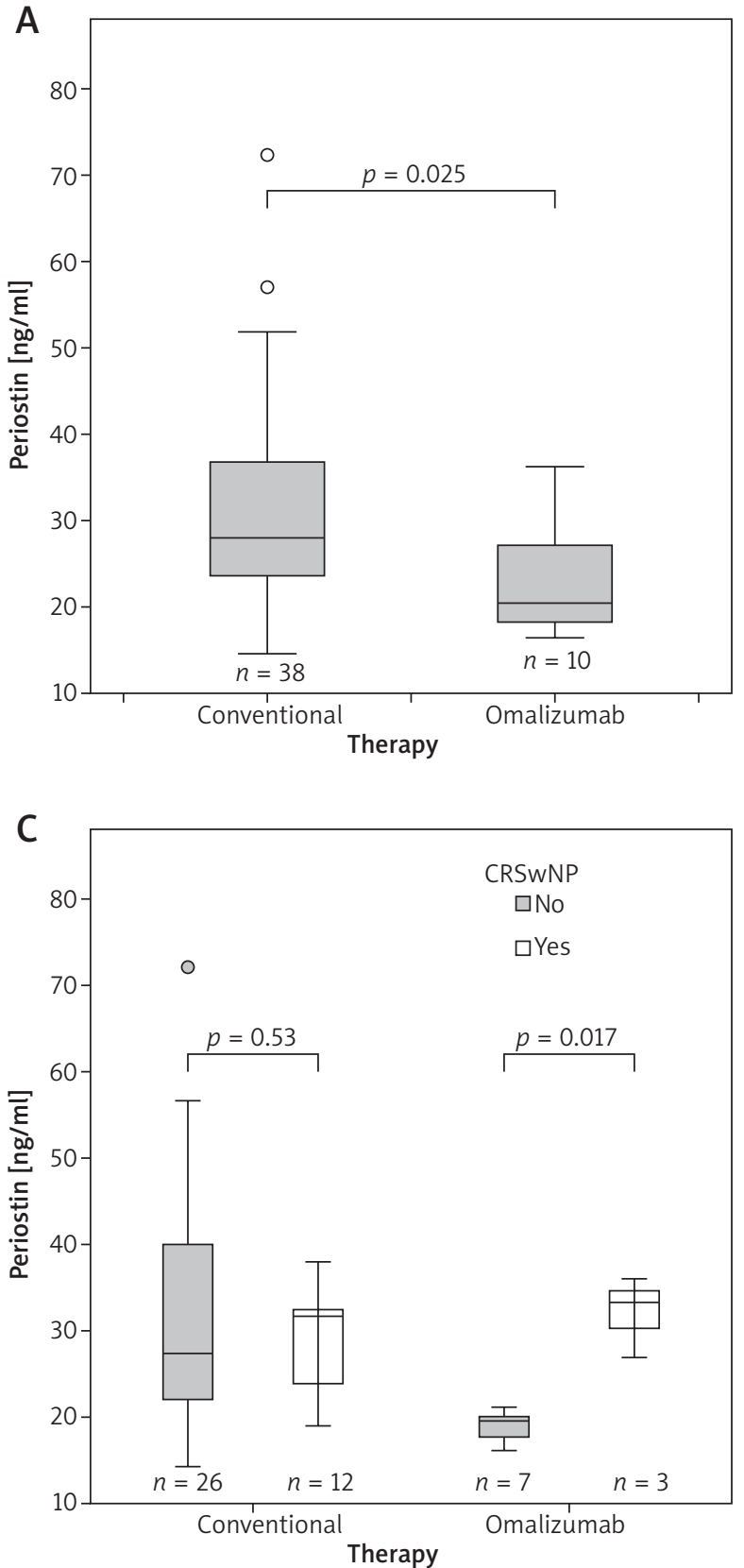

B

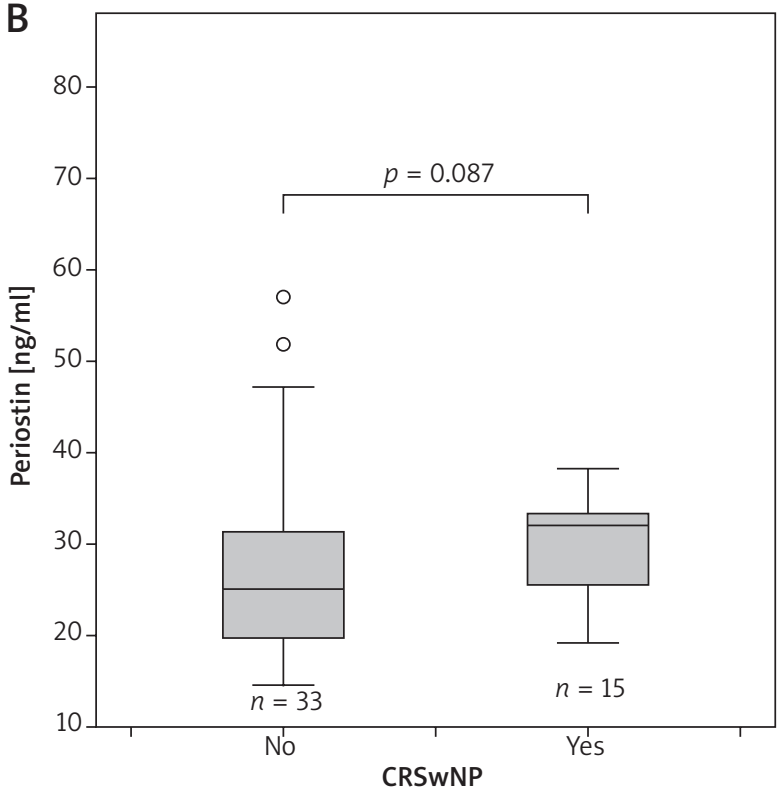

D

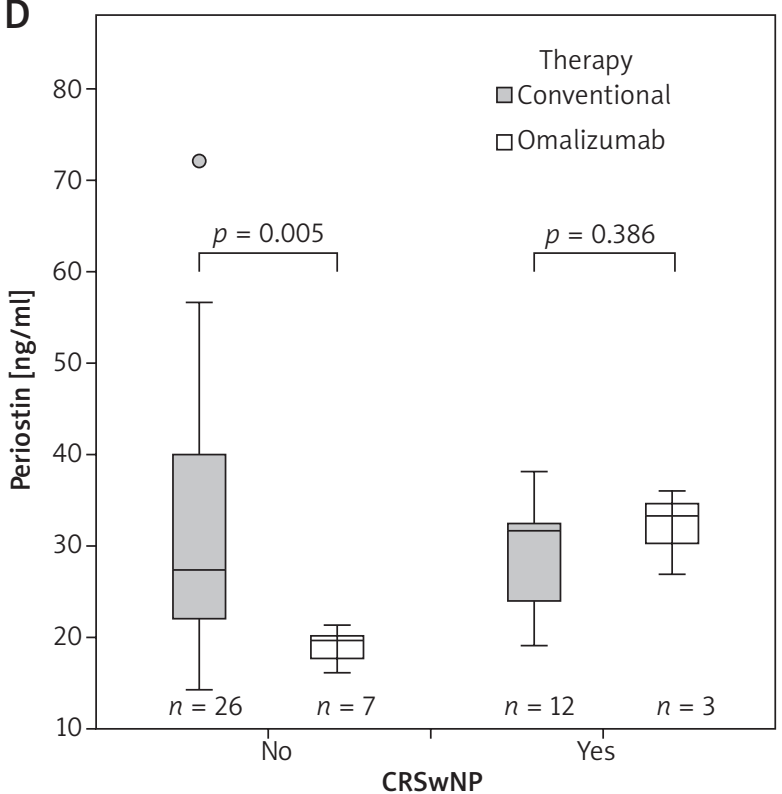

Figure 1. Comparison of periostin levels in particular study subpopulations of particular interest. Serum periostin levels were generally decreased in omalizumab-treated asthma patients in comparison to conventionally treated patients $(p=$ $0.025)(A)$, but there was no overall difference in periostin levels between patients with/without CRSwNP $(p=0.087)(B)$. We confirmed a significant reduction in periostin levels in patients without concurrent CRSwNP only in the omalizumabtreated subgroup $(p=0.017)$ in contrast to patients treated by conventional therapy $(p=0.530)(C)$. Conversely, lower levels of serum periostin in omalizumab-treated patients were discernible only if CRSwNP was not present $(p=0.005)$, in contrast to patients with CRSwNP $(p=0.386)(D)$ (General Linear model, Mann-Whitney $U$ test)

of serum periostin with total IgE levels (Spearman's rho $=0.364 ; p=0.025)$ in conventionally treated patients, with AEC (Spearman's rho $=0.401 ; p=0.021$ ) and eos\% (Spearman's rho $=0.396 ; p=0.023$ ) in patients without CRSWNP and a negative correlation with height (Spearman's rho $=-0.667 ; p=0.007$ ) in patients with CRSwNP.
We have also registered a lack of cross-correlation between periostin levels and monthly OMA dose, cumulative OMA dose and treatment duration in the OMA treatment group. The whole study sample, as well as subpopulations with or without CRSwNP, were excluded from this analysis due to the bias caused 
Table 3. Differences between subgroups of patients according to therapy modality or presence of CRSwNP

\begin{tabular}{|c|c|c|c|c|c|c|c|c|c|c|}
\hline \multirow[t]{4}{*}{ Parameter } & \multicolumn{5}{|c|}{ Therapy } & \multicolumn{5}{|c|}{ CRSwNP } \\
\hline & \multirow{2}{*}{\multicolumn{2}{|c|}{$\begin{array}{c}\text { Conventional } \\
\qquad N=38\end{array}$}} & \multirow{2}{*}{\multicolumn{2}{|c|}{$\begin{array}{c}\text { Omalizumab } \\
\qquad N=10\end{array}$}} & \multirow[t]{3}{*}{$P$-value } & \multirow{2}{*}{\multicolumn{2}{|c|}{$\begin{array}{c}\text { No } \\
N=33\end{array}$}} & \multirow{2}{*}{\multicolumn{2}{|c|}{$\begin{array}{c}\text { Yes } \\
N=15\end{array}$}} & \multirow[t]{3}{*}{$P$-value } \\
\hline & & & & & & & & & & \\
\hline & Median & IQR & Median & IQR & & Median & IQR & Median & IQR & \\
\hline Periostin [ng/ml] & 28.03 & 13.1 & 20.3 & 8.9 & 0.025 & 25.13 & 11.9 & 32.17 & 9.1 & 0.087 \\
\hline $\operatorname{lgE}[\mathrm{IU} / \mathrm{ml}]$ & 206.00 & 286.00 & 398.5 & 1004.00 & 0.491 & 217.00 & 589.3 & 181.00 & 286.00 & 0.982 \\
\hline FeNO(50) [ppb] & 24.00 & 38.00 & 37.5 & 26.00 & 0.99 & 29.00 & 33.00 & 24.00 & 42.00 & 0.973 \\
\hline eos \% & 3.00 & 5.8 & 4.00 & 4.2 & 0.774 & 2.8 & 3.7 & 5.2 & 7.3 & 0.449 \\
\hline $\mathrm{AEC}[\mathrm{eos} / \mathrm{l}]$ & 285.00 & 520.00 & 325.00 & 350.00 & 0.644 & 240.00 & 390.00 & 400.00 & 560.00 & 0.034 \\
\hline $\mathrm{ECP}[\mu \mathrm{g} / \mathrm{l}]$ & 17.835 & 18.67 & 20.915 & 16.27 & 0.965 & 17.55 & 18.57 & 19.77 & 15.72 & 0.29 \\
\hline $\mathrm{FEV}_{1}[\mathrm{l}]$ & 2.19 & 1.04 & 2.32 & 0.46 & 0.397 & 2.265 & 0.985 & 2.015 & 0.94 & 0.17 \\
\hline $\mathrm{FEV}_{1}(\% \mathrm{nh})$ & 68.4 & 37.7 & 74.9 & 24.3 & 0.573 & 79.00 & 41.2 & 60.00 & 26.8 & 0.119 \\
\hline
\end{tabular}

Mann-Whitney $U$ test.

by generally higher periostin levels in patients without OMA. Correlation analysis results are reviewed in Table 4.

\section{Discussion}

The evolving understanding of asthma pathophysiology (in the prism of inflammation paradigm), as well as new therapeutic approaches including biologicals, instigate a further investigation to establish more precise asthma diagnosis and classification. Pathogenesisassociated biomarkers in this particular view bring invaluably helpful information, especially if interpreted not separately, but rather in defined panels (or composite molecular signatures) [36] aimed to describe treatable traits of asthma, like eosinophilic inflammation. This approach is already implemented into our everyday routine [19] but requires very careful assessment of all confounding variables with the potential to bias the result.

Measurement of serum periostin in asthma patients was introduced to broader clinical practice in 2011 by Corren et al. [9], who stated that "periostin-high" patients are better responding to the treatment by lebrikizumab. However, it has been a long venture since its discovery in 1993 [1] through its first detection in subepithelial fibrosis [6], as well as its first association with Th2 driven inflammation in asthma patients [5]. In contrast to other type 2-high eosinophilic inflammation biomarkers, periostin production may putatively contain additional information touching lung tissue fibrosis and remodelling [37], leading among others probably to fixed airway obstruction $[38,39]$ and thus answering slightly different clinical questions considering asthma pathogenesis. These unique features of serum periostin may result from a distinct and very complex network of signalling pathways leading to its elevated production (for example in response to stimulation by transforming growth factor $\beta$
(TGF- $\beta$ ), fibroblast growth factor (FGF), epidermal growth factor (EGF) [40] or IL-13, not exclusively related to atopic reactivity) [12]. Despite very promising potential of serum periostin analysis in asthma patients, this assay still lacks a broadly accepted standardization and thus results coming from different studies using different methods for periostin measurement with varying potencies are not easy to compare and to generalize [25].

To interpret the periostin levels correctly, we inevitably need to identify a majority if not all of other clinically relevant factors and their interactions, influencing its expression. In the case of bronchial asthma patients, it is especially an effect of treatment and an effect of comorbidities. In this regard, periostin has been extensively studied to identify its association with corticosteroid treatment. It has been demonstrated that periostin production can be influenced by corticosteroids in a cell type-dependent manner [41]. Also, a reduction in periostin production reflecting inhaled corticosteroids treatment in asthma patients may reflect a reduction in airway inflammation [14].

Conversely, the persisting elevation of serum periostin despite a high-dose inhaled corticosteroids treatment may pose a risk of airflow limitation due to steroid resistance [39]. The interference of serum periostin levels with biological treatment has also been demonstrated. High baseline serum periostin levels can predict better treatment response to lebrikizumab [9], and at least in part to OMA $[10,11]$. However, in contrast to corticosteroids, we have only scarce evidence of reduction in serum periostin levels reflecting OMA treatment [15].

In addition to treatment, some comorbidities have a particularly pertinent effect on periostin levels. It has been demonstrated that periostin can be recognized as a biomarker of comorbid chronic rhinosinusitis in patients suffering from severe asthma [16] and plays an important role in atopic dermatitis [18, 42, 43]. More- 
over, periostin production can discriminate asthma patients suffering from NSAID hypersensitivity [44] and it reveals a weak though significant negative correlation with BMI [45].

In our work, we aimed to investigate the most important cofactors which need to be taken into account for a more precise interpretation of serum periostin measurement in asthma patients. We focused especially on the mutual interaction between treatment modality (conventional or biological treatment) and comorbidities. We concur with the results of Fingleton et al. [23] stating that serum periostin exerts only a weak cross-correlation with other type-2 biomarkers. In our study, we observed an isolated positive correlation between periostin and total IgE levels in the conventional therapy group and between periostin and AEC or eos\%, respectively, in patients without CRSWNP. A plausible explanation of this result may be only limited statistical collinearity between these biomarkers, given by different biological relevance, which instigate a need for their complex evaluation as a composite signature. We also analysed mutual relationships between periostin levels and anthropometry, and intriguingly, we registered a negative correlation between periostin and height in the CRSwNP subgroup (Table 4), which is in contrast to findings of Kimura et al. [45].

We measured significantly lower periostin levels in patients treated by biological therapy in contrast to those treated conventionally. This result is in line with Caminati et al. [15]. Although we did not perform repeated measures, we suggest that this result reflects the biological potential of OMA to reduce periostin production in pulmonary tissue, as we did not observe any other significant difference between these two groups in terms of the presence of other comorbidities, anthropometry or other characteristics. Moreover, serum periostin was the only Type 2- high inflammation biomarker differing between these two study groups (Table 3), thus underscoring its distinct value.

We attempted to prove the significance of other comorbidities on serum periostin expression. In this regard, we confirmed in line with Asano et al. [16] the significantly higher periostin levels in patients suffering from CRSWNP but not CRSSNP. However, this effect was observable only in a subgroup of patients treated by OMA. Conversely, OMA exerted its retreating potential on periostin production only in a subgroup of patients without CRSwNP (Figure 1). The plausible explanation of this finding may be that allergic patients suffering from CRSWNP constitute a pathogenetically slightly different disease entity (probably more prone to ILC2 orchestrated (ILC-high) inflammation phenotype), despite their allergic history. In this subgroup of patients, periostin production may be more dependent on signalling pathways differing from the conventional atopic reactivity network (for example IL-5 or TGF- $\beta$, see above). These pathways are probably lesser influenced by OMA therapy. Nevertheless,
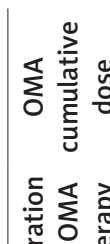

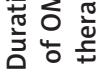

ํํㅁ

$\sum^{\infty}$

$\sum_{0}$

窟

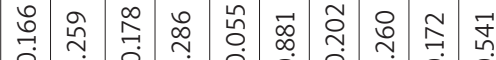

- $\infty$ a $m$ a 0 n

豈

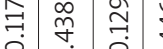

윤 은

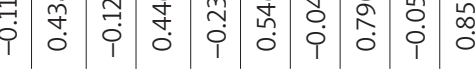

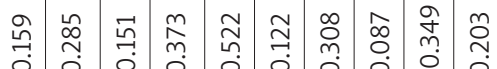

๙0

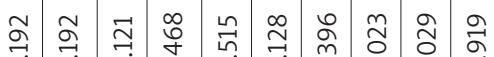

○े

岁

$<$

₹.

200

芯

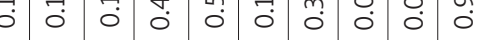

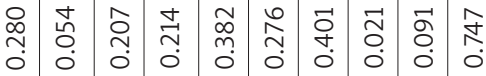

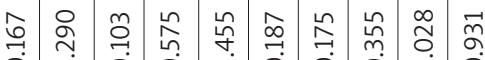

$\sum_{\infty}$

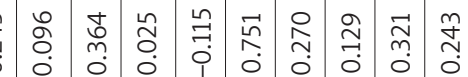

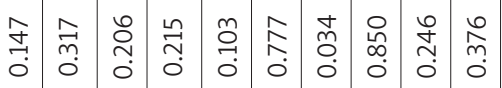

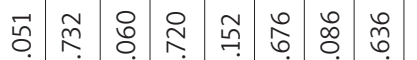

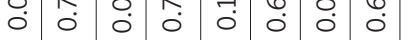

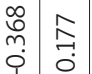

సิ

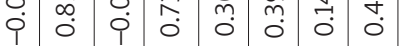

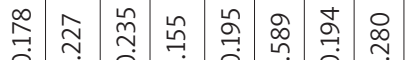

\begin{tabular}{ll}
1 & 1 \\
0 & 0 \\
\hline & 0 \\
0 & 0
\end{tabular}

实

$n$
2
0
0

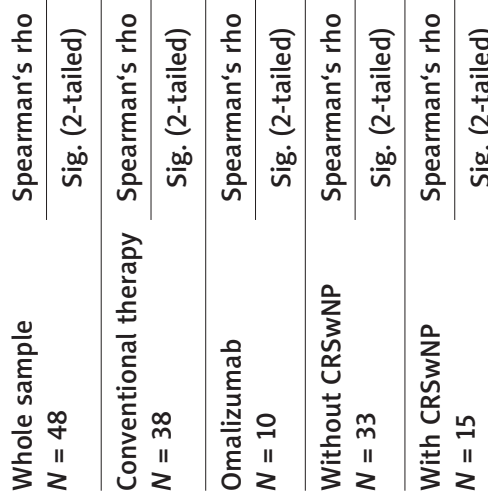

[|ш/8u] u!̣so!̣әd 
this cohort of patients still reveals a clinical benefit from OMA therapy [46]. No other biomarker of eosinophilic inflammation seemed to be able to unravel this particular subpopulation.

We fully admit the weaknesses of our study, especially in terms of its cross-sectional design and low sample size leading to the low power of null hypothesis significance testing. However, this fact, together with a robust analytical approach applied, on the other hand, can unravel only clinically relevant results with high effect size.

\section{Conclusions}

We have observed reproducible changes of serum periostin levels in asthma patients in response to therapy modality (OMA vs. conventional) and presence of CRSWNP. We assume that periostin measurement may bring some new information, especially if assessed with caution and concurrently with other biomarkers of type 2-high inflammation. We bring some new evidence about the mutual interaction between therapy modality and presence of CRSWNP and its effect on periostin production. The weakness of our pilot study is its cross-sectional (not longitudinal) design, low sample size, and heterogeneity of included asthma patients, thus lowering statistical power and generalizability of our outcomes. However, we believe that we can highlight some new important aspects and future directions of periostin measurement and interpretation.

\section{Acknowledgments}

The authors would like to thank Prof. Petr Panzner, MD, PhD, Charles University in Prague, Faculty of Medicine in Pilsen, for generous manuscript revision and helpful recommendations for its final version.

This work was supported by grant PROGRESS Q40 dedicated by the Charles University, Faculty of Medicine in Hradec Králové, Czech Republic.

\section{Conflict of interest}

The authors declare no conflict of interest.

\section{References}

1. Takeshita S, Kikuno R, Tezuka KI, et al. Osteoblast-specific factor 2: cloning of a putative bone adhesion protein with homology with the insect protein fasciclin I. Biochem J 1993; 294: 271-8.

2. Horiuchi K, Amizuka N, Takeshita S, et al. Identification and characterization of a novel protein, periostin, with restricted expression to periosteum and periodontal ligament and increased expression by transforming growth factor beta. J Bone Miner Res 1999; 14: 1239-49.

3. Conway SJ, Izuhara K, Kudo Y, et al. The role of periostin in tissue remodeling across health and disease. Cell Mol Life Sci 2014; 71: 1279-88.
4. Kenji Izuhara A, Conway SJ, Moore BB, et al. Roles of periostin in respiratory disorders. Am J Respir Crit Care Med 2016; 193: 949-56.

5. Woodruff PG, Modrek B, Choy DF, et al. T-helper type 2-driven inflammation defines major subphenotypes of asthma. Am J Respir Crit Care Med 2009; 180: 388-95.

6. Takayama G, Arima K, Kanaji T, et al. Periostin: a novel component of subepithelial fibrosis of bronchial asthma downstream of IL-4 and IL-13 signals. J Allergy Clin Immunol 2006; 118: 98-104.

7. Jia G, Erickson RW, Choy DF, et al. Periostin is a systemic biomarker of eosinophilic airway inflammation in asthmatic patients. J Allergy Clin Immunol 2012; 130: 647-54.

8. Woodruff PG, Boushey HA, Dolganov GM, et al. Genomewide profiling identifies epithelial cell genes associated with asthma and with treatment response to corticosteroids. Pnas 2007; 104: 15858-63.

9. Corren J, Lemanske RF, Hanania NA, et al. Lebrikizumab treatment in adults with asthma. N Engl J Med 2011; 365: 1088-98.

10. Hanania NA, Wenzel S, Roseń K, et al. Exploring the effects of omalizumab in allergic asthma: an analysis of biomarkers in the EXTRA study. Am J Respir Crit Care Med 2013; 187: 804-11.

11. Tajiri T, Matsumoto H, Gon Y, et al. Utility of serum periostin and free IgE levels in evaluating responsiveness to omalizumab in patients with severe asthma. Allergy 2016; 71: 1472-9.

12. Matsumoto H. Serum periostin: a novel biomarker for asthma management. Allergol Int 2014; 63: 153-60.

13. Izuhara K, Ohta SS, Ono J. Using periostin as a biomarker in the treatment of asthma. Allergy Asthma Immunol Res 2016; 8: 491-8.

14. Hoshino M, Ohtawa J, Akitsu K. Effect of treatment with inhaled corticosteroid on serum periostin levels in asthma. Respirology 2016; 21: 297-303.

15. Caminati M, Gatti D, Dama A, et al. Serum periostin during omalizumab therapy in asthma: a tool for patient selection and treatment evaluation. Ann Allergy Asthma Immunol 2017; 119: 460-2.

16. Asano T, Kanemitsu Y, Takemura M, et al. Serum periostin as a biomarker for comorbid chronic rhinosinusitis in patients with asthma. Ann Am Thorac Soc 2017; 14: 667-75.

17. Ishida A, Ohta N, Suzuki Y, et al. Expression of pendrin periostin in allergic rhinitis chronic rhinosinusitis. Allergol Int 2012; 61: 589-95.

18. Kou K, Okawa T, Yamaguchi Y, et al. Periostin levels correlate with disease severity and chronicity in patients with atopic dermatitis. Br J Dermatol 2014; 171: 283-91.

19. Terl M, Sedlak V, Cap P, et al. Asthma management: a new phenotype-based approach using presence of eosinophilia and allergy. Allergy 2017; 72: 1279-87.

20. Katial RK, Bensch GW, Busse WW, et al. Changing paradigms in the treatment of severe asthma: the role of biologic therapies. J Allergy Clin Immunol Pract 2017; 5: S1-14.

21. Brusselle GG, Maes T, Bracke KR. Eosinophilic airway inflammation in nonallergic asthma. Nat Med 2013; 19: 977-9.

22. Godar M, Blanchetot C, de Haard H, et al. Personalized medicine with biologics for severe type 2 asthma: current status and future prospects. MAbs 2018; 10: 34-45.

23. Fingleton J, Braithwaite I, Travers J, et al. Serum periostin in obstructive airways Disease. Eur Respir J 2016; 47: 1383-91. 
24. Bossuyt PM, Reitsma JB, Bruns DE, et al. STARD 2015: An updated list of essential items for reporting diagnostic accuracy studies. BMJ 2015; 351: h5527.

25. Arron JR, Izuhara K. Asthma biomarkers: what constitutes a "gold standard"? Thorax 2015; 70: 105-7.

26. FitzGerald M, Bateman ED, Boulet LP, et al. Global strategy for asthma management and prevention (GINA 2015). 2015.

27. Bousquet J, Rabe K, Humbert M, et al. Predicting and evaluating response to omalizumab in patients with severe allergic asthma. Respir Med 2007; 101: 1483-92.

28. Hamilton RG, Marcotte GV, Saini SS. Immunological methods for quantifying free and total serum IgE levels in allergy patients receiving Omalizumab (Xolair) therapy. I Immunol Methods 2005; 303: 81-91.

29. Pellegrino R, Viegi G, Brusasco V, et al. Interpretative strategies for lung function tests. Eur Respir J 2005; 26: 948-68.

30. Miller MR, Hankinson J, Brusasco V, et al. Standardisation of spirometry. Eur Respir J 2005; 26: 319-38.

31. Dweik RA, Boggs PB, Erzurum SC, et al. An official ATS clinical practice guideline: Interpretation of exhaled nitric oxide levels (FENO) for clinical applications. Am J Respir Crit Care Med 2011; 184: 602-15.

32. Reddel HK, Taylor DR, Bateman ED, et al. An official American Thoracic Society/European Respiratory Society statement: asthma control and exacerbations - standardizing endpoints for clinical asthma trials and clinical practice. Am J Respir Crit Care Med 2009; 180: 59-99.

33. Akdis CA, Akdis M, Bieber T, et al. Diagnosis and treatment of atopic dermatitis in children and adults: European Academy of Allergology and Clinical Immunology/American Academy of Allergy, Asthma and Immunology/PRACTALL consensus report. Allergy 2006; 61: 969-87.

34. Fokkens RW, Lund V, Mullol J, et al. EPOS European Position Paper on Rhinosinusitus and Nasal Polyps 2012. Rhinol Suppl 2012: 1-298.

35. Melillo G, Balzano G, Bianco S, et al. Report of the INTERASMA working group on standardization of inhalation provocation tests in aspirin-induced asthma: oral and inhalation provocation tests for the diagnosis of aspirin-induced asthma. Allergy 2001; 56: 899-911.

36. Wagener AH, Yick CY, Brinkman P, et al. Toward composite molecular signatures in the phenotyping of asthma. Ann Am Thorac Soc 2013; 10 Suppl: S197-205.

37. Uchida M, Shiraishi H, Ohta S, et al. Periostin, a matricellular protein, plays a role in the induction of chemokines in pulmonary fibrosis. Am J Respir Cell Mol Biol 2012; 46: 677-86.

38. Takahashi K, Meguro K, Kawashima H, et al. Serum periostin levels serve as a biomarker for both eosinophilic airway inflammation and fixed airflow limitation in well-controlled asthmatics. J Asthma 2018; 56: 236-43.

39. Kanemitsu Y, Matsumoto H, Izuhara K, et al. Increased periostin associates with greater airflow limitation in patients receiving inhaled corticosteroids. J Allergy Clin Immunol 2013; 132: 305-12..

40. Morra L, Moch H. Periostin expression and epithelial-mesenchymal transition in cancer: a review and an update. Virchows Arch 2011; 459: 465-75.

41. Shoda T, Futamura K, Kobayashi F, et al. Cell type-dependent effects of corticosteroid on periostin production by primary human tissue cells. Allergy 2013; 68: 1467-70.

42. Masuoka M, Shiraishi H, Ohta S, et al. Periostin promotes chronic allergic inflammation in response to Th2 cytokines. J Clin Invest 2012; 122: 2590-600.
43. Shiraishi H, Masuoka M, Ohta S, et al. Periostin contributes to the pathogenesis of atopic dermatitis by inducing TSLP production from keratinocytes. Allergol Int 2012; 61: 563-72.

44. Kim MAA, Kyung Yoon M, Lee YSS, et al. Clinical implication of the serum periostin level for differentiating phenotypes of NSAID hypersensitivity. Allergol Int 2016; 65: 492-4.

45. Kimura H, Konno S, Makita H, et al. Serum periostin is associated with body mass index and allergic rhinitis in healthy and asthmatic subjects. Allergol Int 2018; 67: 357-63.

46. Gevaert P, Calus L, Van Zele T, et al. Omalizumab is effective in allergic and nonallergic patients with nasal polyps and asthma. J Allergy Clin Immunol 2013; 131 110-6.e1. 